\title{
UNIT SUM NUMBERS OF RIGHT SELF-INJECTIVE RINGS
}

\author{
Dinesh Khurana and Ashish K. Srivastava
}

In a recent paper (which is to appear in J. Algebra Appl.) we proved that every element of a right self-injective ring $R$ is a sum of two units if and only if $R$ has no factor ring isomorphic to $\mathbb{Z}_{2}$ and hence the unit sum number of a nonzero right self-injective ring is $2, \omega$ or $\infty$. In this paper we characterise right self-injective rings with unit sum numbers $\omega$ and $\infty$. We prove that the unit sum number of a right self-injective ring $R$ is $\omega$ if and only if $R$ has a factor ring isomorphic to $\mathbb{Z}_{2}$ but no factor ring isomorphic to $\mathbb{Z}_{2} \times \mathbb{Z}_{2}$, and also in this case every element of $R$ is a sum of either two or three units. It follows that the unit sum number of a right self-injective ring $R$ is $\infty$ precisely when $R$ has a factor ring isomorphic to $\mathbb{Z}_{2} \times \mathbb{Z}_{2}$. We also answer a question of Henriksen (which appeared in J. Algebra, Question E, page 192), by giving a large class of regular right self-injective rings having the unit sum number $\omega$ in which not all non-invertible elements are the sum of two units.

We shall consider associative rings with identity. Our modules will be unital right modules with endomorphisms acting on the left.

A ring $R$ is said to have the $n$-sum property, for a positive integer $n$, if each of its elements can be written as a sum of exactly $n$ units of $R$. It is obvious that a ring having the $n$-sum property also has the $k$-sum property for every positive integer $k>n$. The unit sum number of a ring $R$, denoted by usn $(R)$, is the least integer $n$, if it exists, such that $R$ has the $n$-sum property. If $R$ has an element which is not a sum of units then we set usn $(R)$ to be $\infty$, and if every element of $R$ is a sum of units but $R$ does not have the $n$-sum property for any $n$, then we set $\operatorname{usn}(R)=\omega$. Clearly, usn $(R)=1$ if and only if $R$ has only one element. The unit sum number of a module $M$, denoted by usn $(M)$, is the unit sum number of its endomorphism ring. The topic has been studied extensively (see $[2,3,5,6,7,10,11,15,17,19,20])$.

In 1954 Zelinsky [20] proved that usn $\left(V_{D}\right)=2$, where $V$ is a vector space over a division ring $D$, unless $\operatorname{dim}(V)=1$ and $D=\mathbb{Z}_{2}$, in which case $\operatorname{usn}\left(V_{D}\right)=3$. As $\operatorname{End}\left(V_{D}\right)$ is a (von Neumann) regular ring, in 1958 Skornyakov ([16, Problem 31, p. 167]), asked: Is every element of a regular ring a sum of units? This question was settled by

Received 28th August, 2006

This work was done when the first author was visiting the Center of Ring Theory and its Applications, Ohio University, and he is highly grateful to Professor S. K. Jain for the invitation and hospitality.

Copyright Clearance Centre, Inc. Serial-fee code: 0004-9727/07 \$A2.00+0.00. 
Bergman (see [9]) in the negative who gave an example of a regular directly-finite ring $R$ in which 2 is invertible such that $\operatorname{usn}(R)=\infty$. The result of Zelinsky has also generated a considerable interest in the unit sum numbers of right self-injective rings as $\operatorname{End}\left(V_{D}\right)$ is right self-injective (see $[17, \mathbf{1 9}, \mathbf{1 5}]$ ). Recently, the authors in $[13]$ have characterised right self-injective rings with unit sum number 2 , thereby generalising the result of Zelinsky. It is proved in [13] that for a right self-injective ring $R, \operatorname{usn}(R)=2$ if and only if the identity of $R$ is a sum of two units, if and only if $R$ has no factor ring isomorphic to $\mathbb{Z}_{2}$. So it is immediate that the unit sum number of a nonzero right self-injective ring is $2, \omega$ or $\infty$. In this paper we prove that the right self-injective rings with unit sum number $\omega$ are precisely the rings which have a factor ring isomorphic to $\mathbb{Z}_{2}$ but no factor ring isomorphic to $\mathbb{Z}_{2} \times \mathbb{Z}_{2}$. It follows that a right self-injective ring has the unit sum number $\infty$ if and only if it has a factor ring isomorphic to $\mathbb{Z}_{2} \times \mathbb{Z}_{2}$. In ([10, Question E, p. 192]) Henriksen asked if there exists a regular ring $R$ with $\operatorname{usn}(R) \neq \infty$ which has a non-invertible element that cannot be written as a sum of two units. We answer this question by giving a large class of regular right self-injective rings having the unit sum number $\omega$ such that not all non-invertible elements are the sum of two units. However, we prove that if $R$ is a regular right self-injective ring with usn $(R) \neq \infty$ then every non-invertible element in $R$ is a sum of either two or three units.

The work depends heavily on the theory of types of regular right self-injective rings introduced by Kaplansky [12]. While writing the paper we received the sad news of the demise of Professor Kaplansky. We dedicate this paper to the memory of Professor Kaplansky.

For terms not defined we refer the reader to [4]. The following two lemmas are inherent in [13] but we include them here for the sake of completeness.

LEMмA 1. For any nonzero regular right self-injective ring $R, \operatorname{usn}\left(M_{2}(R)\right)=2$. In particular, for any purely infinite regular right self-injective ring $R, \operatorname{usn}(R)=2$.

Proof: By ([1, Corollary 2.6]) every $A \in M_{2}(R)$ admits a diagonal reduction, that is, there exist invertible matrices $P$ and $Q$ in $M_{2}(R)$ such that $P A Q$ is a diagonal matrix, say $\left(\begin{array}{ll}a & 0 \\ 0 & b\end{array}\right)$. Then $P A Q=\left(\begin{array}{ll}a & 0 \\ 0 & b\end{array}\right)=\left(\begin{array}{ll}a & 1 \\ 1 & 0\end{array}\right)+\left(\begin{array}{cc}0 & -1 \\ -1 & b\end{array}\right)$ is sum of two units and so $A$ is a sum of two units.

If $R$ is purely infinite regular right self-injective ring, then $R_{R} \cong(R \oplus R)_{R}$ implying that, as rings, $R \cong M_{2}(R)$ and so the result follows from above.

Using Lemma 1 we obtain the following.

LEMMA 2. A nonzero regular right self-injective ring $R$ has a ring decomposition $R=S \times T$ where usn $(S)=1$ or 2 and $T$ is an Abelian regular right self-injective ring.

Proof: $\mathrm{By}$ ([4, Proposition 10.21]) $R \cong R_{1} \times R_{2}$, where $R_{1}$ is either zero or purely infinite and $R_{2}$ is directly finite. By Lemma 1 , usn $\left(R_{1}\right)=1$ or 2 . By ([4, Theorem 10.22]) $R_{2} \cong R_{3} \times R_{4}$, where $R_{3}$ is Type $I_{f}$ and $R_{4}$ is Type $I I_{f}$. Further, by ([4, Theorem 
$10.24]), R_{3} \cong \prod M_{n}\left(S_{i}\right)$, where each $S_{i}$ is an Abelian regular right self-injective ring. Also, as $R_{4}$ has no nonzero Abelian idempotents, there exists an idempotent $e \in R_{4}$ such that $\left(R_{4}\right)_{R_{4}} \cong\left(e R_{4} \oplus e R_{4}\right)_{R_{4}}$ (see [4, Proposition 10.28]), implying that, as rings, $R_{4} \cong M_{2}\left(e R_{4} e\right)$. Thus $R \cong R_{1} \times\left(\prod M_{n}\left(S_{i}\right)\right) \times M_{2}\left(e R_{4} e\right)$. We let $S=R_{1} \times\left(\prod_{n>1} M_{n}\left(S_{i}\right)\right)$ $\times M_{2}\left(e R_{4} e\right)$ and $T=\prod S_{i}$. By Lemma 1 usn $(S)=1$ or 2 .

Lemma 3. (Vámos [19, Proposition 19]) Let $M_{R}$ be a nonsingular injective module. Then $\operatorname{End}\left(M_{R}\right)$ is a Boolean ring if and only if the identity endomorphism of no direct summand of $M$ is a sum of two units.

LEMma 4. ([13, Theorem 1]) For a nonzero right self-injective ring $R$, the following conditions are equivalent:

(1) $\operatorname{usn}(R)=2$.

(2) The identity of $R$ is a sum of two units.

(3) $R$ has no factor ring isomorphic to $\mathbb{Z}_{2}$.

LEMma 5. Let $T$ be an Abelian regular right self-injective ring. Then $T=T_{1} \times T_{2}$ where usn $\left(T_{1}\right)=1$ or 2 and $T_{2}$ is either zero or a Boolean ring.

Proof: In view of Lemma 4 it is enough to show that $T$ has a ring decomposition $T=T_{1} \times T_{2}$, where $T_{2}$ is a Boolean ring and the identity in $T_{1}$ is a sum of two units in $T_{1}$. We shall prove this using a standard Zorn's lemma argument as used in ([19, Lemma 17]). Let $\mathcal{T}$ be the set of all pairs of the form $(A, u)$ where $A$ is a submodule of $T_{T}$ and $u$ is an automorphism of $A$ such that $I_{A}-u$ is also an automorphism of $A$, where $I_{A}$ is the identity automorphism of $A$. Clearly $(0,0) \in \mathcal{T}$. Then $\mathcal{T}$ has an obvious partial order, that is, $(A, u) \leqslant\left(A^{\prime}, u^{\prime}\right)$ if $A \subseteq A$ and $u^{\prime}$ agrees with $u$ on $A$. Also $\mathcal{T}$ is easily seen to be inductive and so by Zorn's lemma $\mathcal{T}$ has a maximal element $\left(T_{1}, v\right)$ say. If $T_{1}$ is not injective, then $v$ extends to an automorphism $v^{\prime}$ of an injective hull $E\left(T_{1}\right)$ of $T_{1}$ in $T_{T}$, and so $\left(E\left(T_{1}\right), v^{\prime}\right) \in \mathcal{T}$ violating the maximality of $\left(T_{1}, v\right)$. Thus $T_{1}$ is injective and so $T=T_{1} \oplus T_{2}$ for some submodule $T_{2}$ of $T_{T}$. As $\left(T_{1}, v\right)$ is a maximal element of $\mathcal{T}$, it is clear that the identity endomorphism of no direct summand of $T_{2}$ is a sum of two units. So, by Lemma 3, $\operatorname{End}_{T}\left(T_{2}\right)$ is a Boolean ring. As every idempotent in $T$ is central, the module decomposition $T_{T}=T_{1} \oplus T_{2}$ gives us a ring decomposition $T=T_{1} \times T_{2}$ with $\operatorname{End}_{T}\left(T_{2}\right) \cong T_{2}$ a Boolean ring. Also the identity in $T_{1}$ is a sum of two units in $T_{1}$.

We know that a right self-injective ring is right continuous (see [4]). The following example shows that Lemma 5 is not true even for commutative regular continuous rings. Let $F$ be a field having $\mathbb{Z}_{2}$ as a proper subfield. Set $F_{n}=F$ and $K_{n}=\mathbb{Z}_{2}$ for each positive integer $n$. Let

$$
R=\left\{\left(x_{n}\right)_{\mathbb{N}} \in \prod_{\mathbf{N}} F_{n}: x_{n} \in K_{n} \text { for all but finitely many } n\right\} .
$$

Then $R$ is a commutative continuous ring (see [4, Example 13.8]) which clearly is regular. It is easy to see that the identity of $R$ is not a sum of two units. An element $\left(x_{n}\right)_{N}$ of $R$ 
is an idempotent precisely when every component is either 0 or 1 and so it is clear that $e R$ is not a Boolean ring for any idempotent $e \in R$. Also the element $(1,0,1,0, \ldots)$ is not a sum of units in $R$ implying that usn $(R)=\infty$.

The following result characterises the regular right self-injective rings with various unit sum numbers.

TheOREM 6 . The unit sum number of a nonzero regular right self-injective ring $R$ is 2 , $\omega$ or $\infty$. Moreover,

(1) $\operatorname{usn}(R)=2$ if and only if $R$ has no (nonzero) Boolean ring as a ring direct summand.

(2) $\operatorname{usn}(R)=\omega$ if and only if $R$ has $\mathbb{Z}_{2}$, but no Boolean ring with more than two elements, as a ring direct summand. Moreover, in this case every non-invertible element of $R$ is a sum of either two or three units.

(3) $\operatorname{usn}(R)=\infty$ if and only if $R$ has a Boolean ring with more than two elements as a ring direct summand.

PROOF: In view of Lemma 2 and Lemma $5, R=R_{1} \times B$ where usn $\left(R_{1}\right)=1$ or 2 and $B$ is a Boolean ring. It is clear that the unit sum number of a nonzero Boolean ring is $\infty$ unless it is isomorphic to $\mathbb{Z}_{2}$, in which case the unit sum number is $\omega$. So (1) is immediate. If usn $(R) \neq 2$, then $B \neq 0$. Clearly, usn $(R)=\omega$ if and only if $B \cong \mathbb{Z}_{2}$ and usn $(R)=\infty$ if and only if $B$ has more than two elements. Also if usn $(R)=\omega$, then $R \cong R_{1} \times \mathbb{Z}_{2}$ and it is clear than any non-invertible element of $R$ is a sum of either two or three units.

In ([10, Question E, p. 192]) Henriksen asked if there is a regular ring, with every element the sum of units, in which there are non-invertible elements that are not the sum of two units. The following example answers this question.

EXAMPLE 7. Let $S$ be a nonzero regular right self-injective ring which does not have a factor ring isomorphic to $\mathbb{Z}_{2}$. For instance, take $S$ to be any field other than $\mathbb{Z}_{2}$. Let $R=S \times \mathbb{Z}_{2}$. Clearly $R$ is a regular right self-injective ring and, by Theorem 6 , the usn $(R)=\omega$. But the element $(0,1)$ of $R$ is a non-unit which cannot be written as a sum of two units.

We are now ready to prove our main result.

THEOREM 8. The unit sum number of a nonzero right self-injective ring $R$ is $2, \omega$ or $\infty$. Moreover,

(1) $\operatorname{usn}(R)=2$ if and only if $R$ has no factor ring isomorphic to $\mathbb{Z}_{2}$.

(2) $\operatorname{usn}(R)=\omega$ if and only if $R$ has a factor ring isomorphic to $\mathbb{Z}_{2}$, but has no factor ring isomorphic to $\mathbb{Z}_{2} \times \mathbb{Z}_{2}$. In this case every non-invertible element of $R$ is a sum of either two or three units.

(3) $\operatorname{usn}(R)=\infty$ if and only if $R$ has a factor ring isomorphic to $\mathbb{Z}_{2} \times \mathbb{Z}_{2}$. 
Proof: We know that $R / J(R)$ is a nonzero regular right self-injective ring (see [18]) and also it is clear that usn $(R)=\operatorname{usn}(R / J(R))$. So the result follows from Theorem 6 and the fact that any Boolean ring with more than two elements has a factor ring isomorphic to $\mathbb{Z}_{2} \times \mathbb{Z}_{2}$.

As defined above, the unit sum number of a module is the unit sum number of its endomorphism ring. So the unit sum number of any module, whose endomorphism ring modulo the jacobson radical is right self-injective, is $2, \omega$ or $\infty$. We list two such classes of modules below.

COROLlaRY 9. Let $M_{R}$ be a quasi-injective or a flat cotorsion module. Then $\operatorname{usn}(M)$ is 2 , $\omega$ or $\infty$.

Proof: Let $E=\operatorname{End}_{R}(M)$. Then $E / J(E)$ is a regular right self-injective ring (see $[14,8])$. So the result follows from Theorem 8 .

We have seen that if $R$ is a regular right self-injective ring with usn $(R) \neq \infty$ then every non-invertible element is a sum of either two or three units. This makes us ask the following question.

QUESTION 10. Let $R$ be a regular ring with usn $(R) \neq \infty$. Is every non-invertible element a sum of two or three units?

The following question of Henriksen also seems to be open.

QUESTION 11. (Henriksen [10, p. 192]) Let $R$ be a regular ring in which 2 is invertible such that $\operatorname{usn}(R) \neq \infty$. Is every non-invertible element of $R$ a sum of two units?

In view of Theorem 6 the above question has a positive answer for regular right self-injective rings.

\section{REFERENCES}

[1] P. Ara, K.R. Goodearl, K. C. O'Meara and E. Pardo, 'Diagonalization of matrices over regular rings', Linear Algebra Appl. 265 (1997), 147-163.

[2] N. Ashrafi and P. Vámos, 'On the unit sum number of some rings', Quart. J. Math. 56 (2005), 1-12.

[3] W. Fisher, R. L Snider, 'Rings generated by their units', J. Algebra 42 (1976), 363-368.

[4] K.R. Goodearl, von Neumann regular rings (Krieger Publishing Company, Malabar, FL, 1991).

[5] B. Goldsmith, C. Meehan and S. L Wallutis, 'On unit sum numbers of rational groups', Proceedings of the Second Honolulu Conference on Abelian Groups and Modules (Honolulu, HI, 2001), Rocky Mountain J. Math. 32 (2002), 1431-1450.

[6] R. Göbel and A. Opdenhövel, 'Every endomorphism of a local Warfield module of finite torsion-free rank is the sum of two automorphisms', J. Algebra 233 (2000), 758-771.

[7] B. Goldsmith, S. Pabst and A. Scott, 'Unit sum numbers of rings and modules', Quart. J. Math. Oxford Ser. (2) 49 (1998), 331-344.

[8] P.A. Guil Asensio and I. Herzog, 'Left cotorsion ring', Bull. London Math Soc. 36 (2004), 303-309. 
[9] D. Handelman, 'Perspectivity and cancellation in regular rings', J. Algebra 48 (1977), 1-16.

[10] M. Henriksen, 'Two classes of rings generated by their units', J. Algebra 31 (1974), 182-193.

[11] P. Hill, 'Endomorphism rings generated by units', Trans. Amer. Math. Soc. 141 (1969), 99-105.

[12] I. Kaplansky, Rings of operators (Benjamin, New York, 1968).

[13] D. Khurana and A. K. Srivastava, 'Right self-injective rings in which every element is sum of two units', J. Algebra Appl. (to appear).

[14] B.L. Osofsky, 'Endomorphism rings of quasi-injective modules', Canad. J. Math. 20 (1968), 895-903.

[15] R. Raphael, 'Rings which are generated by their units', J. Algebra 28 (1974), 199-205.

[16] L.A. Skornyakov, Complemented modular lattices and regular rings (Oliver Boyd, Edinburgh, 1964).

[17] Y. Utumi, 'On continuous regular rings and semisimple self-injective rings', Canad. J. Math. 12 (1960), 597-605.

[18] Y. Utumi, 'Self-injective rings', J. Algebra 6 (1967), 56-64.

[19] P. Vámos, '2-good rings', Quart. J. Math. 56 (2005), 417-430.

[20] D. Zelinsky, 'Every linear transformation is a sum of nonsingular ones', Proc. Amer. Math. Soc. 5 (1954), 627-630.

Department of Mathematics

Panjab University

Chandigarh-160014

India

e-mail: dkhurana@pu.ac.in
Department of Mathematics

Ohio University

Athens, OH 45701

United States of America

e-mail: ashish@math.ohiou.edu 